\title{
Re-irradiation for recurrent or second primary head and neck
}

\section{cancer}

\author{
Hye In Lee ${ }^{1}$, Jin Ho Kim ${ }^{1}$, Soon-Hyun Ahn², Eun-Jae Chung ${ }^{2}$, Bhumsuk Keam³ ${ }^{3}$ Keun-Yong Eom ${ }^{4}$, Woo-Jin Jeong ${ }^{5}$, \\ Ji-Won $\mathrm{Kim}^{6}$, Chan Woo Wee ${ }^{7}$, Hong-Gyun Wu $\mathrm{Wu}^{1,8,9}$ \\ ${ }^{1}$ Department of Radiation Oncology, Seoul National University Hospital, Seoul National University College of Medicine, Seoul, Korea \\ ${ }^{2}$ Department of Otorhinolaryngology-Head and Neck Surgery, Seoul National University Hospital, Seoul National University College of Medicine, \\ Seoul, Korea \\ ${ }^{3}$ Department of Internal Medicine, Seoul National University Hospital, Seoul National University College of Medicine, Seoul, Korea \\ ${ }^{4}$ Department of Radiation Oncology, Seoul National University Bundang Hospital, Seoul National University College of Medicine, Seongnam, \\ Korea \\ ${ }^{5}$ Department of Otorhinolaryngology-Head and Neck Surgery, Seoul National University Bundang Hospital, Seoul National University College of \\ Medicine, Seongnam, Korea \\ ${ }^{6}$ Department of Internal Medicine, Seoul National University Bundang Hospital, Seoul National University College of Medicine, Seongnam, Korea \\ ${ }^{7}$ Department of Radiation Oncology, Seoul Metropolitan Government Seoul National University Boramae Medical Center, Seoul, Korea \\ ${ }^{8}$ Cancer Research Institute, Seoul National University College of Medicine, Seoul, Korea \\ ${ }^{9}$ Institute of Radiation Medicine, Medical Research Center, Seoul National University, Seoul, Korea
}

Received: June 23, 2021

Revised: August 7, 2021

Accepted: August 17, 2021

Correspondence:

Hong-Gyun Wu

Department of Radiation Oncology,

Seoul National University College of

Medicine, 101 Daehak-ro, Jongno-gu,

Seoul 03080, Korea

Tel: +82-2-2072-3177

E-mail:wuhg@snu.ac.kr

ORCID:

https://orcid.org/0000-0002-0167-7991
Purpose: To investigate the efficacy and safety of intensity-modulated radiotherapy (IMRT)-based re-irradiation (reRT) for recurrent or second primary head and neck cancer (HNC).

Materials and Methods: Patients who underwent IMRT-based reRT for recurrent or second primary HNC between 2007 and 2019 at two institutions were included. Medical records and dosimetric data were retrospectively reviewed. Overall survival (OS), progression-free survival (PFS), severe late toxicities, and clinicopathological prognostic factors were analyzed.

Results: A total of 42 patients were analyzed. With a median follow-up of 15.1 months (range, 3.7 to 85.8 months), the median OS was 28.9 months with a 2 -year OS rate of $54.6 \%$. The median PFS and 2-year PFS rates were 10.0 months and 30.9\%, respectively. Multivariate analysis showed that good performance (Eastern Cooperative Oncology Group [ECOG] 0 or 1), a longer time interval $(\geq 24$ months) between radiotherapy courses, and higher reRT dose (>60 Gy) were significantly favorable factors for OS (all $p<0.05$ ). Higher reRT dose and salvage surgery were significantly associated with improved PFS (all $p<0.05$ ). Regarding the Multi-Institution Reirradiation (MIRI) Collaborative RPA classification, the 2-year OS rates of each class were $87.5 \%$ in class I, $51.8 \%$ in class II, and $0 \%$ in class III ( $p=0.008)$. Grade $\geq 3$ late toxicity was reported in $10(23.8 \%)$ patients. There was no significant factor associated with increased late toxicities.

Conclusion: IMRT-based reRT should be considered as a treatment option for patients with recurrent or second primary HNC. Further trials are needed to establish a subset of patients who may benefit from reRT without severe late toxicity.

Keywords: Head and neck cancer, Re-irradiation, Intensity-modulated radiotherapy, Overall survival, Progression-free survival, Toxicity

Copyright (C) 2021 The Korean Society for Radiation Oncology

This is an Open Access article distributed under the terms of the Creative Commons Attribution Non-Commercial License (http://creativecommons.org/licenses/by-nc/4.0/) which permits unrestricted non-commercial use, distribution, and reproduction in any medium, provided the original work is properly cited. 


\section{Introduction}

Despite multimodality treatments for head and neck cancer (HNC), almost half of the patients still experience locoregional recurrence or second primary cancer [1-3]. Recurrent or second primary HNC not only affects prognosis but also decreases the quality of life. Salvage surgery for locoregional recurrence is considered a primary curative treatment, with a 5-year survival rate approaching 40\% [4]. However, radical resection is not possible in most patients, and it could be accompanied by substantial complications and worsen the quality of life. Re-irradiation (ReRT) has been considered as another curative treatment option for recurrent or second primary HNC; however, increased risk of severe or life-threatening treatment-related toxicity and tumor radioresistance pose challenges to reRT.

For the last decade, intensity-modulated radiotherapy (IMRT) has become the standard radiation technique for the treatment of patients with HNC. IMRT has demonstrated the ability to reduce toxicity and improve disease control, compared with conventional radiotherapy (RT) and three-dimensional conformal radiotherapy (3D-CRT) $[5,6]$. IMRT-based reRT has led to the expectation that the efficacy and safety after reRT would be improved, and has contributed to an increasing application of reRT in clinical practice. Although several studies have reported the outcomes of IMRT-based reRT for HNC, the exact role of reRT remains unclear.

In this study, we evaluated the treatment outcomes, toxicities, and prognostic factors in patients who received IMRT-based reRT for recurrent or second primary HNC in two affiliated institutions.

\section{Materials and Methods}

We retrospectively reviewed patients who received IMRT-based reRT for recurrent or second primary HNC between January 2007 and December 2019 at two institutions: Seoul National University Hospital and Seoul National University Bundang Hospital. The inclusion criteria were as follows: (1) adult patients aged $\geq 18$ years who had locoregional recurrent or second primary HNC and completed IMRT-based reRT as planned; (2) the overlapping volume that received $\geq 45$ Gy for initial irradiation also received $\geq 45 \mathrm{~Gy}$ for reRT; and (3) availability of a medical record and dosimetric data related to both initial and secondary radiation profiles. No limit was placed on the time interval between the RT courses. Patients treated with stereotactic body RT or those initially diagnosed with distant metastasis or other malignancies were excluded. Patients may have received salvage surgery or chemotherapy, as indicated. Finally, 42 patients with recurrent $(n=35)$ or second primary $(n=7)$ HNC were included. We classified as a second primary cancer if the recurrent tumor occurred $\geq 5$ years after initial diagnosis or histologically different despite the overlapping volume. This study was approved by the Institutional Review Board of Seoul Nataional University Hospital and Seoul National University Bundang Hospital (No. H-1803-106-932). The requirement for informed consent was waived. We carried out this research according to the principles expressed in the Declaration of Helsinki.

All patients received IMRT-based reRT at 1.8 to 3 Gy per fraction, once daily, and five times a week. The treatment protocol was as follows: 67.5 Gy in 30 fractions for curative aim, 63 Gy in 28 fractions for postoperative aim. The total dose of reRT was lowered if the cumulative dose of organ-at-risk (such as brainstem, optic chiasm/nerve, or spinal cord) exceeded dose contraints, or the patient had poor performance status to tolerate the full dose. Dose constraints of organs at risk were a maximum of $45 \mathrm{~Gy}$ for the spinal cord, $54 \mathrm{~Gy}$ for the brainstem, optic chiasm and optic nerve, and $\mathrm{V}_{20}<50 \%$ for the parotid gland, with an assumption of 50\% dose tolerance recovery for a retreatment interval $\geq 12$ months.

Overall survival (OS) was defined as the time from the start date of reRT to the date of death or the last date of clinical follow-up. Progression-free survival (PFS) was defined as the time from the start date of reRT to any documented recurrence at the local site, regional lymph node, or distant site. Acute and late toxicities were graded using the Common Terminology Criteria for Adverse Events version 4.0. Acute toxicity was defined as toxicity observed during the RT period or within 6 months from the end date of reRT. Late toxicity was defined as toxicity developing more than 6 months after the end date of reRT. Events developing after disease progression were considered disease-related symptoms and were not included in the toxicity events. Additionally, we divided patients into three prognostic subgroups using the recursive partitioning analysis (RPA) defined by the Multi-Institution Reirradiation (MIRI) Collaborative: class I included patients $>2$ years from their initial course of RT with resected tumors; class II included patients $>2$ years with unresected tumors or those $\leq 2$ years and without organ dysfunction (feeding tube or tracheostomy dependence), and the remaining patients formed class III. We assessed survival outcomes of each RPA class [7].

The OS and PFS rates were estimated using the Kaplan-Meier method, with differences assessed using the log-rank test. Potential prognostic factors associated with OS and PFS were investigated using univariate and multivariate Cox proportional hazards models. Logistic regression analyses were employed to determine the associations between prognostic factors and late toxicities. Statistical significance for hypothesis testing was assumed to be 0.05 . All statistical analyses were performed by STATA version 16.0. (StataCorp, College Station, TX, USA). 


\section{Results}

\section{Patients and treatment characteristics}

Between January 2007 and December 2019, 42 patients from two institutions were included. Table 1 presents the patients, disease, and treatment characteristics of the study population. The median age at initial RT was 57 years (range, 26 to 76 years) and 61 years (range, 28 to 80 years) at reRT. Thirty-four patients (81.0\%) had squamous cell carcinoma, and 29 (69.0\%) had stage III-IV disease at initial diagnosis. The most common primary subsite was the nasopharynx (35.7\%), followed by the sinonasal cavity (21.4\%).

Salvage surgery preceded reRT in 17 (40.5\%) patients. Of the

Table 1. Baseline characteristics $(n=42)$

\begin{tabular}{|c|c|c|}
\hline Characteristic & & Value \\
\hline \multirow[t]{18}{*}{ Initial presentation } & Age (yr) & $57(26-76)$ \\
\hline & Sex & \\
\hline & Male & $31(73.8)$ \\
\hline & Female & $11(26.2)$ \\
\hline & Performance status (ECOG) & \\
\hline & $0-1$ & 39 (92.8) \\
\hline & $2-3$ & $3(7.2)$ \\
\hline & Histology & \\
\hline & $\mathrm{SqCC}$ & $34(81.0)$ \\
\hline & Non-SqCC & $8(19.0)$ \\
\hline & Primary subsite & \\
\hline & Nasopharynx & $15(35.7)$ \\
\hline & Sinonasal & $9(21.4)$ \\
\hline & Oral cavity & $4(9.5)$ \\
\hline & Larynx & $4(9.5)$ \\
\hline & Hypopharynx & $4(9.5)$ \\
\hline & Salivary gland & $4(9.5)$ \\
\hline & Oropharynx & $2(4.8)$ \\
\hline \multirow[t]{8}{*}{ Initial treatment } & Surgery & \\
\hline & No & $26(61.9)$ \\
\hline & Yes & $16(38.1)$ \\
\hline & Chemotherapy & \\
\hline & No & $16(38.1)$ \\
\hline & Yes & $26(61.9)$ \\
\hline & RT dose (Gy) & $67.5(54-72)$ \\
\hline & RT fractional dose (Gy) & $2.25(1.8-2.7)$ \\
\hline \multirow[t]{6}{*}{ Second presentation } & Age (yr) & $61(28-80)$ \\
\hline & $<60$ & $18(42.9)$ \\
\hline & $\geq 60$ & $24(57.1)$ \\
\hline & Performance status (ECOG) & \\
\hline & $0-1$ & $34(81.0)$ \\
\hline & $2-3$ & $8(19.0)$ \\
\hline
\end{tabular}

postoperative patients, seven (41.2\%) underwent R0 resection and 10 (58.8\%) underwent R1-2 resection. Simultaneous chemotherapy was administered to 25 patients (59.5\%) with cisplatin as the most commonly used systemic agent ( $n=18 ; 72 \%$ ). Twenty patients (47.6\%) underwent reRT with curative aim, 17 patients (40.5\%) with postoperative aim, and five (11.9\%) with palliative aim. Thirty-three patients (78.6\%) received reRT to the involved field, defined as the gross tumor or surgical bed plus a margin of $0.5-1.0$ $\mathrm{cm}$, and nine patients (21.4\%) received reRT to an elective field encompassing the gross tumor or surgical bed with an elective nodal area. The median interval between initial and secondary RT courses was 24.1 months (range, 4.6 to 116.6 months). Regarding RPA

\begin{tabular}{|c|c|c|}
\hline Characteristic & & Value \\
\hline \multirow[t]{14}{*}{ Second presentation } & Presentation type & \\
\hline & Recurrent & 35 (83.3) \\
\hline & Second primary & $7(16.7)$ \\
\hline & Failure type & \\
\hline & Local failure & $21(50.0)$ \\
\hline & Regional failure & $5(11.9)$ \\
\hline & Locoregional failure & $9(21.4)$ \\
\hline & Recurrent tumor size $(\mathrm{cm})$ & \\
\hline & $<3$ & $26(61.9)$ \\
\hline & $\geq 3$ & $16(38.1)$ \\
\hline & RPA class & \\
\hline & Class I & $10(23.8)$ \\
\hline & Class II & $28(66.7)$ \\
\hline & Class III & $4(9.5)$ \\
\hline \multirow[t]{17}{*}{ Second treatment } & Surgery & \\
\hline & No & $25(59.5)$ \\
\hline & Yes & $17(40.5)$ \\
\hline & Chemotherapy & \\
\hline & No & $17(40.5)$ \\
\hline & Yes & $25(59.5)$ \\
\hline & ReRT dose (Gy) & $63(45-67.5)$ \\
\hline & $\leq 60$ & $18(42.9)$ \\
\hline & $>60$ & $24(57.1)$ \\
\hline & ReRT fractional dose (Gy) & $2.25(1.8-3.0)$ \\
\hline & Treatment volume of reRT & \\
\hline & Involved field & $33(78.6)$ \\
\hline & Elective field & $9(21.4)$ \\
\hline & Cumulative RT dose (Gy) & $128.4(104.4-139.5)$ \\
\hline & $\begin{array}{l}\text { The interval between RT } \\
\text { courses (mo) }\end{array}$ & $24.1(4.6-116.6)$ \\
\hline & $<24$ & $21(50.0)$ \\
\hline & $\geq 24$ & $21(50.0)$ \\
\hline
\end{tabular}

Values are presented as median (range) or number (\%). ECOG, Eastern Cooperative Oncology Group; SqCC, squamous cell carcinoma; RT, radiotherapy; RPA, recursive partitioning analysis; reRT, re-irradiation. 
classes, classes I, II, and III accounted for 23.8\%, 66.7\%, and 9.5\%, respectively.

\section{Overall survival and prognostic factors}

The median follow-up time from the start date of reRT was 15.1 months for all patients (range, 3.7 to 85.8 months) and 21.2 months for survivors (range, 7.6 to 64.8 months). Seventeen patients (40.5\%) remained alive at the time of the last observation. The median OS was 28.9 months (range, 3.7 to 85.8 months). The 1-year and 2-year OS rates were $63.3 \%$ (95\% confidence interval [Cl], 46.6\%-65.1\%) and $54.6 \%$ (95\% Cl, 37.8\%-68.7\%), respectively. The 2-year OS rate was $75.1 \%$ (95\% Cl, 46.3\%-89.9\%) for patients who received postoperative RT and 33.3\% (95\% Cl, 14.3\%-53.7\%) for patients who received definitive $\mathrm{RT}$. Among postoperative patients, the 2-year OS rate was $85.7 \%(95 \% \mathrm{Cl}, 33.4 \%-97.8 \%)$ in patients undergoing $\mathrm{RO}$ resection, and $66.7 \%(95 \% \mathrm{Cl}, 27.2 \%-88.2 \%)$ in patients undergoing $\mathrm{R} 1$ or $\mathrm{R} 2$ resection. Fig. $1 \mathrm{~A}$ presents $\mathrm{OS}$ stratified by salvage surgery for recurrent tumors.

Table 2 presents the univariate and multivariate Cox proportional hazards models for OS. On univariate analysis, good performance (Eastern Cooperative Oncology Group [ECOG] 0 or 1), the longer time interval between RT courses ( $\geq 24$ months), higher reRT dose (>60 Gy), and salvage surgery were associated with improved OS. Of these, good performance status, the longer time interval between RT courses, and higher reRT dose were significantly associated with the improved OS on multivariate analysis (all $p<0.05$ ).

\section{Progression-free survival and prognostic factors}

The median PFS was 10.0 months (range, 1.3 to 85.8 months). The
1- and 2-year PFS rates were 40.9\% (95\% Cl, 25.6\%- 55.6\%) and $30.9 \%$ (95\% Cl, 16.6\%-46.3\%), respectively. The 1-year PFS rate was $70.6 \%$ for postoperative patients (95\% Cl, 43.2\%-86.5\%) and 17.0\% (95\% Cl, 4.6\%-36.0\%) for definitive patients. Fig. 1B presents PFS stratified by salvage surgery for recurrent tumors. During the follow-up period, a total of 27 patients (64.3\%) showed disease progression, of which 17 (40.5\%), 8 (19.0\%), and 7 (16.7\%) had local, regional, and distant failures, respectively. Two patients (4.8\%) had mucosal recurrence. The 1- and 2-year local control (LC) rates were 57.5\% (95\% Cl, 39.8\%-71.7\%) and 41.8\% (95\% Cl, 24.2\%$58.6 \%)$, respectively.

In our Cox proportional hazards model for PFS, a longer time interval between RT courses, higher reRT dose, and salvage surgery exhibited significant associations on univariate analysis. On multivariate analysis, higher reRT dose and salvage surgery were significantly associated with improved PFS (all $p<0.05$ ).

\section{Survival validation according to MIRI-RPA classes}

All patients were classified into three RPA classes defined by MIRI collaboration according to the time interval between RT courses, salvage surgery, and organ dysfunction, as follows: those with interval $>2$ years between RT courses with resected tumors (class I, $n=10)$, those with interval $>2$ years between RT courses with unresected tumors or with interval $\leq 2$ years between RT courses without organ dysfunction (class II, $n=28$ ), and those with interval $\leq 2$ years between RT courses with organ dysfunction (class III, $\mathrm{n}=4)$. Fig. 2 presents the Kaplan-Meier curves stratified by the RPA class. The 2-year OS rates were $87.5 \%$ in class $1,51.8 \%$ in class $\mathrm{II}$, and $0 \%$ in class $\mathrm{III}(\mathrm{p}=0.008)$.

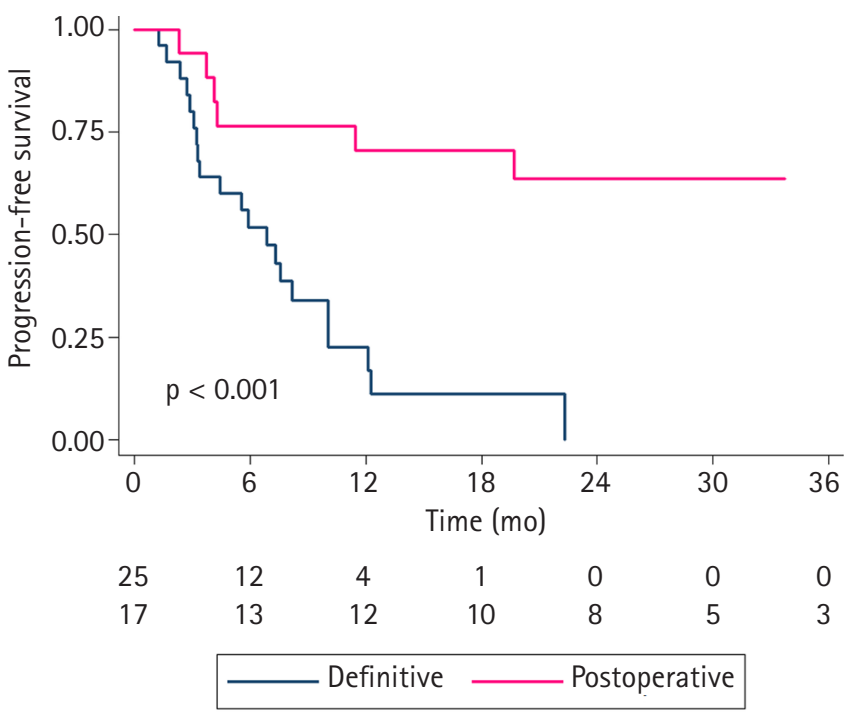

Fig. 1. Kaplan-Meier curve of overall survival (A) and progression-free survival (B) according to salvage surgery. 
Table 2. Univariate and multivariate analysis affecting overall survival

\begin{tabular}{|c|c|c|c|c|c|c|}
\hline \multirow{2}{*}{ Variable } & \multicolumn{3}{|c|}{ Univariate } & \multicolumn{3}{|c|}{ Multivariate } \\
\hline & $\mathrm{HR}$ & $95 \% \mathrm{Cl}$ & $p$-value & $\mathrm{HR}$ & $95 \% \mathrm{Cl}$ & $p$-value \\
\hline \multicolumn{7}{|l|}{ Age at reRT (yr) } \\
\hline$<60$ & 1.000 & & & & & \\
\hline$\geq 60$ & 1.322 & $0.592-2.949$ & 0.495 & & & \\
\hline \multicolumn{7}{|l|}{ Sex } \\
\hline Male & 1.000 & & & & & \\
\hline Female & 1.809 & $0.770-4.251$ & 0.189 & & & \\
\hline \multicolumn{7}{|c|}{ Performance status (ECOG) } \\
\hline $0-1$ & 1.000 & & & 1.000 & & \\
\hline $2-3$ & 2.947 & $1.236-7.021$ & 0.022 & 1.857 & $0.700-4.919$ & 0.005 \\
\hline \multicolumn{7}{|l|}{ Histology } \\
\hline $\mathrm{SqCC}$ & 1.000 & & & & & \\
\hline Non-SqCC & 0.730 & $0.246-2.163$ & 0.557 & & & \\
\hline \multicolumn{7}{|l|}{ Primary subsite } \\
\hline Nasopharynx & 1.000 & & & & & \\
\hline Sinonasal & 2.698 & $0.833-8.733$ & 0.098 & & & \\
\hline Oral cavity & 14.616 & $3.597-59.387$ & $<0.001$ & & & \\
\hline Larynx & 0.820 & $0.097-6.889$ & 0.855 & & & \\
\hline Hypopharynx & 2.811 & $0.528-14.974$ & 0.226 & & & \\
\hline Salivary gland & 2.361 & $0.572-9.741$ & 0.235 & & & \\
\hline Oropharynx & 5.988 & $1.108-32.359$ & 0.038 & & & \\
\hline \multicolumn{7}{|l|}{ Presentation type } \\
\hline Recurrent & 1.000 & & & & & \\
\hline Second primary & 0.328 & $0.076-1.404$ & 0.080 & & & \\
\hline \multicolumn{7}{|l|}{ Failure type } \\
\hline Local failure & 1.000 & & & & & \\
\hline Regional failure & 2.475 & $0.764-8.013$ & 0.130 & & & \\
\hline Locoregional failure & 1.755 & $0.654-4.711$ & 0.264 & & & \\
\hline \multicolumn{7}{|c|}{ Recurrent or second primary tumor size $(\mathrm{cm})$} \\
\hline$<3$ & 1.000 & & & & & \\
\hline$\geq 3$ & 1.291 & $0.573-2.909$ & 0.539 & & & \\
\hline \multicolumn{7}{|c|}{ The interval between RT courses (mo) } \\
\hline$<24$ & 1.000 & & & 1.000 & & \\
\hline$\geq 24$ & 0.304 & $0.128-0.717$ & 0.005 & 0.333 & $0.124-0.892$ & 0.029 \\
\hline \multicolumn{7}{|l|}{ ReRT dose (Gy) } \\
\hline$\leq 60$ & 1.000 & & & 1.000 & & \\
\hline$>60$ & 0.161 & $0.063-0.412$ & 0.000 & 0.194 & $0.064-0.587$ & 0.004 \\
\hline \multicolumn{7}{|l|}{ Treatment volume of reRT } \\
\hline Involved field & 1.000 & & & & & \\
\hline Elective field & 1.166 & $0.433-3.132$ & 0.764 & & & \\
\hline \multicolumn{7}{|l|}{ Salvage surgery } \\
\hline No & 1.000 & & & 1.000 & & \\
\hline Yes & 0.359 & $0.141-0.910$ & 0.021 & 0.569 & $0.217-1.490$ & 0.251 \\
\hline \multicolumn{7}{|l|}{ Chemotherapy } \\
\hline No & 1.000 & & & & & \\
\hline Yes & 1.263 & $0.550-2.896$ & 0.581 & & & \\
\hline \multicolumn{7}{|l|}{ MIRI RPA class } \\
\hline Class I & 1.000 & & & & & \\
\hline Class II & 4.531 & $1.044-19.659$ & 0.044 & & & \\
\hline Class III & 11.794 & $2.065-67.346$ & 0.006 & & & \\
\hline
\end{tabular}




\section{Toxicity}

The severe late toxicity related to reRT is presented in Table 3 . There was no grade $\geq 3$ acute toxicity. Grade $\geq 3$ late toxicity was observed in 10 patients (23.8\%), which consisted of osteonecrosis (n $=4)$, severe bleeding $(n=2)$, dysphagia $(n=1)$, cellulitis $(n=1)$, laryngitis $(n=1)$, and airway stricture $(n=1)$. All patients with grade $\geq 3$ late toxicity were hospitalized, and five patients (11.9\%) underwent surgical treatment to repair toxicity. One patient (2.4\%) with an airway stricture was intubated and later died from aspiration pneumonia. There was no significant factor associated with increased grade $\geq 3$ late toxicity in logistic regression analysis.

\section{Discussion and Conclusion}

In this study, we evaluated the treatment outcomes and toxicities

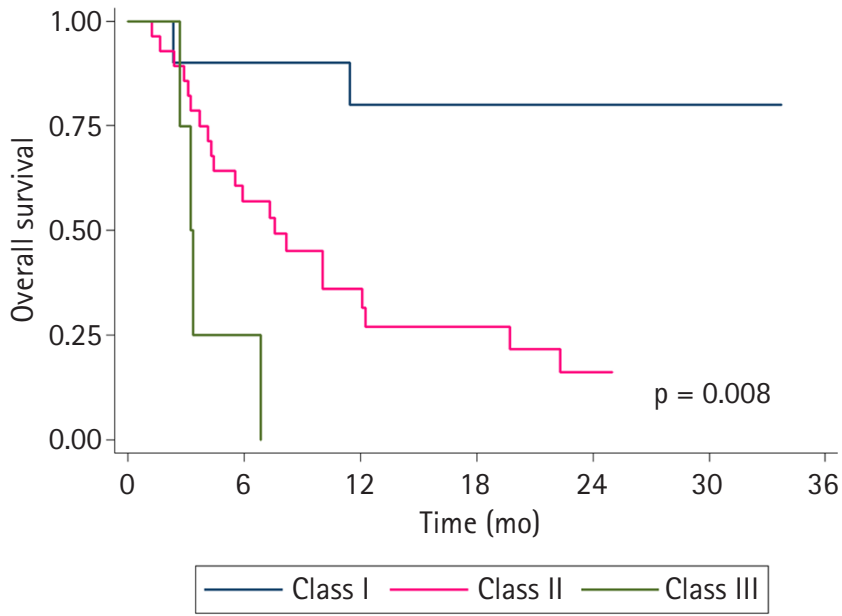

Fig. 2. Kaplan-Meier curve of overall survival according to the recursive partitioning analysis classes defined by the Multi-Institution Reirradiation (MIRI)-Collaborative. of IMRT-based reRT for recurrent or second primary HNC. IMRT-based reRT, which enables accurate targeting of irregularly shaped tumors and minimizing dose exposure to the surrounding normal tissues, has been reported to have more favorable outcomes compared to conventional RT or 3D-CRT. The two landmark prospective reRT trials in the pre-IMRT era, namely, Radiation Therapy Oncology Group (RTOG) 9610 and RTOG 9911 trials, reported 2-year OS rates of $15 \%$ and $25 \%$, respectively $[8,9]$. Among recent studies in the IMRT era, the 2-year OS rate ranged from 33\% to 59\% and the 2-year LC rate ranged from 36\% to 65\% [10-14]. We also demonstrated similar results with 2-year OS and LC rates of $54.6 \%$ and $41.8 \%$, respectively. The treatment outcomes and toxicity data of recent studies are summarized in Table 4 [7,10-13,15-18].

Our study supports salvage surgery as a standard modality for recurrent or second primary HNC, with a 2-year OS rate of $75.1 \%$ for postoperative patients and 33.3\% for definitive patients. Although most patients who received definitive RT had the advanced irresectable disease or were unfit for surgery, there was a signifi-

Table 3. Incidence of severe late toxicity following IMRT-based reirradiation

\begin{tabular}{lc}
\hline Variable & No. $(\%)$ \\
\hline Grade $\geq 3$ late toxicity & $10(8.5)$ \\
Grade 3 & \\
Osteoradionecrosis & $4(9.5)$ \\
Bleeding & $2(4.7)$ \\
Dysphagia & $1(2.3)$ \\
Cellulitis & $1(2.3)$ \\
Laryngitis & $1(2.3)$ \\
Grade 5 & \\
$\quad$ Airway stricture & $1(2.3)$ \\
\hline
\end{tabular}

IMRT, intensity-modulated radiotherapy.

Table 4. Summary of head and neck re-irradiation (reRT) in recent studies

\begin{tabular}{|c|c|c|c|c|c|c|c|c|c|}
\hline \multirow{2}{*}{ Study } & \multirow{2}{*}{$\mathrm{n}$} & \multirow{2}{*}{$\begin{array}{c}\text { Median } \\
\text { follow-up (mo) }\end{array}$} & \multirow{2}{*}{$\begin{array}{l}\text { ReRT dose } \\
\text { (Gy) }\end{array}$} & \multirow{2}{*}{$\begin{array}{l}\text { Resection } \\
\text { rate }(\%)\end{array}$} & \multicolumn{2}{|c|}{ Overall survival } & \multicolumn{2}{|c|}{ Local control } & \multirow{2}{*}{$\begin{array}{l}\text { Grade } \geq 3 \\
\text { late toxicity (\%) }\end{array}$} \\
\hline & & & & & Median (mo) & $2-y r(\%)$ & Median (mo) & $2-y r(\%)$ & \\
\hline Takiar et al. [13] & 227 & 24.7 & 66 & 50.5 & NR & 57.0 & NR & 65.0 & 35.4 \\
\hline \multirow[t]{2}{*}{ Curtis et al. [15] } & 81 & 78.1 & 69.6 (def) & 51.8 & 22.2 & 50.0 & NR & 60.0 & NR \\
\hline & & & 60 (postop) & & & & & & \\
\hline Lee et al. [16] & 66 & 23 & 70 & 37.9 & 22 & 49.0 & 19.8 & 42.0 & 26.7 \\
\hline Velez et al. [17] & 78 & 27.2 & 60 & 40.8 & NR & 51.0 & NR & 36.8 & 32.8 \\
\hline Ohnleiter et al. [18] & 50 & 13.6 & 66 & 80.0 & 15.7 & 33.9 & 8.3 & 35.9 & 28.0 \\
\hline Choi et al. [11] & 73 & 28 & 60 & 38.4 & 33 & 58 & 23 & 36.0 & 22.2 \\
\hline Awan et al. [12] & 45 & 16 & 60 & 73.3 & NR & 45.3 & NR & NR & 17.4 \\
\hline \multirow[t]{2}{*}{ Ward et al. [7] } & 412 & 10.4 & 60 & 47.3 & 16.5 & 40.0 & NR & 54 (def) & 14.2 \\
\hline & & & & & & & & 60 (postop) & \\
\hline Lee et al. [10] & 118 & 18.5 & 59.4 & 40.7 & 20.1 & 43.1 & 28.9 & 53.5 & 8.5 \\
\hline
\end{tabular}

def, definitive radiotherapy; postop, postoperative radiotherapy; NR, not reported. 
cant difference between the two groups. MIRI collaborative reported a 2-year OS rate of 45\% for postoperative patients and 36\% for definitive patients [7]. Bots et al. [14] reported a 2-year OS rate of $48 \%$ and $17 \%$ for postoperative and definitive patients, respectively. A meta-analysis of 17 studies reported that the most commonly reported LC predictor was surgical resection, and the rate of salvage surgery significantly influenced the 2 -year LC rate $(58.5 \%$ vs. $45.9 \% ; p=0.011$ ) [19]. In our multivariate analysis, salvage surgery exhibited a significant association with PFS, but not OS. Salvage surgery is an important factor considering that recurrent HNC is generated from radioresistant clonogens after initial RT [20]. However, surgical resection with clear margins is challenging and must be balanced with the morbidity of the procedure, the functional consequences of organ mutilation, and the likelihood of success [21]. Therefore, salvage surgery should be offered as the standard approach when medically safe and reasonable functional deficits are expected.

Several studies have reported a relationship between higher reRT dose and improved outcomes in patients with recurrent HNC $[22,23]$. Since radioresistance might be induced in recurrent HNC, higher reRT doses or radiosensitizing agents are required to achieve adequate tumor control rates $[24,25]$. In our multivariate analysis, a higher reRT dose (> 60 Gy) was a significant prognostic factor for both OS and PFS, but not for late toxicity. It is recommended to deliver sufficient reRT dose for recurrent or second primary HNC; however, a balance between adequate tumor control and minimal severe toxicity should be considered.

The appropriate time interval between RT courses has not been established; however, many studies have reported that a longer interval between RT courses is associated with improved survival outcomes $[7,17,26]$. In this study, patients who received reRT with a time interval longer than 24 months showed significantly improved OS. The fact that it might be reluctant to provide sufficient reRT dose to patients with a short time interval may have adverse effects on tumor control. In addition, patients with recurrent tumors presenting within a very short interval from initial treatment were considered to have persistent disease. Some authors have proposed a threshold of up to 2 years for resected tumors $[27,28]$. The time interval between RT courses also reflects the biological aggressiveness of HNC. Thus, the time interval between RT courses appears to affect prognosis; however, it should be noted that most relapses are observed within the first 24 months.

Additionally, several other factors correlated with treatment outcomes. The primary site of HNC is an important prognostic factor. Good prognosis has been observed in nasopharyngeal and laryngeal cancer, whereas oral cavity and hypopharyngeal cancers have a relatively poor prognosis [1]. We found that patients with nasopha- ryngeal and laryngeal cancer achieved superior OS, whereas patients with oral cavity cancer showed the worst survival outcomes, followed by hypopharyngeal cancer. The nasopharynx was the most common primary site in our cohort; this probably had a positive effect on the outcomes. Chemotherapy was not associated with treatment outcomes or late toxicity in our analysis. As it is a retrospective analysis, the omission of chemotherapy in unfit patients and the presence of risk factors in patients receiving chemotherapy may bias the results of chemotherapy.

The major concern for head and neck reRT is life-threatening late toxicity. In our study, 10 patients (23.8\%) experienced severe late toxicities and one (2.4\%) died of toxicity. Severe late toxicity has been reported to be between $8 \%$ and 50\% in previous studies [19]. However, these rates may have been underestimated as many patients were censored for disease progression or death. Most patients with local progression in our population had severe dysphagia, pain, or necrosis, which might have been affected by both disease progression and treatment. We evaluated several prognostic factors using logistic regression analyses, and none showed a significant association with severe late toxicity. Ward et al. [29] reported a competing risk nomogram that predicted severe late toxicity after reRT for recurrent or second primary HNC. The nomogram model included initial RT dose, tumor site, organ dysfunction, any surgery, and patient's age. Notably, modifiable treatment-related variables such as the reRT dose or regimen, reRT field, and the use of systemic therapy were not major determinants of late toxicity, which is consistent with our results. Due to the moderate rates of severe toxicity, all patients should be well informed about the benefits and risks of reRT before proceeding.

It is still challenging to identify patients who would benefit most from reRT. The MIRI collaborative suggested the RPA classification according to the time interval between RT courses, salvage surgery, and organ dysfunction [7]. In the report of MIRI collaboration, the 2-year OS rate was 61.9\% in RPA class I, 40.0\% in RPA class II, and $16.8 \%$ in RPA class III. Several studies validated the MIRI-RPA classification, and it was also applicable to our patient population (2year OS rate, $87.5 \%$ vs. $51.8 \%$ vs. $0 \%$ in each class; $p=0.008$ ). The MIRI-RPA classification could be a useful tool for selecting suitable patients for reRT in clinical practice.

There were some limitations to our study. Our sample was small and heterogeneous, with diversity in primary locations, histology, tumor stage, and treatments. Additionally, as this was a retrospective study, the events and toxicities could have been underestimated due to incomplete medical records. Nevertheless, despite these limitations, the current analysis suggested the role of reRT as a treatment option for patients with recurrent or second primary HNC.

In conclusion, IMRT-based reRT for recurrent or second primary 
HNC exhibited improved survival outcomes and moderate severe toxicity. Salvage surgery, sufficient reRT dose, and the longer time interval between RT courses could contribute to more favorable outcomes. Careful patient selection for reRT is important, and MIRI-RPA classification might be a useful tool. Further trials are needed to determine the subset of patients who may benefit from reRT without severe late toxicity.

\section{Conflict of Interest}

No potential conflict of interest relevant to this article was reported.

\section{Acknowledgements}

This work was supported by the National Research Foundation of Korea (NRF) grant funded by the Korea government (Ministry of Science and ICT) (No. 2019R1F1A1040583 and NRF-2020M2D9A 1093990).

\section{References}

1. Kim YS. Reirradiation of head and neck cancer in the era of intensity-modulated radiotherapy: patient selection, practical aspects, and current evidence. Radiat Oncol J 2017;35:1-15.

2. Brockstein B, Haraf DJ, Rademaker AW, et al. Patterns of failure, prognostic factors and survival in locoregionally advanced head and neck cancer treated with concomitant chemoradiotherapy: a 9-year, 337-patient, multi-institutional experience. Ann Oncol 2004;15:1179-86.

3. Cooper JS, Pajak TF, Rubin P, et al. Second malignancies in patients who have head and neck cancer: incidence, effect on survival and implications based on the RTOG experience. Int J Radiat Oncol Biol Phys 1989;17:449-56.

4. Bachar GY, Goh C, Goldstein DP, O'Sullivan B, Irish JC. Long-term outcome analysis after surgical salvage for recurrent tonsil carcinoma following radical radiotherapy. Eur Arch Otorhinolaryngol 2010;267:295-301.

5. Zhang B, Mo Z, Du W, Wang Y, Liu L, Wei Y. Intensity-modulated radiation therapy versus $2 \mathrm{D}-\mathrm{RT}$ or $3 \mathrm{D}-\mathrm{CRT}$ for the treatment of nasopharyngeal carcinoma: a systematic review and meta-analysis. Oral Oncol 2015;51:1041-6.

6. Lee N, Chan K, Bekelman JE, et al. Salvage re-irradiation for recurrent head and neck cancer. Int J Radiat Oncol Biol Phys 2007;68:731-40.

7. Ward MC, Riaz N, Caudell JJ, et al. Refining patient selection for reirradiation of head and neck squamous carcinoma in the IMRT era: a multi-institution cohort study by the MIRI Collaborative. Int J Radiat Oncol Biol Phys 2018;100:586-94.

8. Spencer SA, Harris J, Wheeler RH, et al. Final report of RTOG 9610, a multi-institutional trial of reirradiation and chemotherapy for unresectable recurrent squamous cell carcinoma of the head and neck. Head Neck 2008;30:281-8.

9. Langer CJ, Harris J, Horwitz EM, et al. Phase II study of low-dose paclitaxel and cisplatin in combination with split-course concomitant twice-daily reirradiation in recurrent squamous cell carcinoma of the head and neck: results of Radiation Therapy Oncology Group Protocol 9911. J Clin Oncol 2007;25:4800-5.

10. Lee J, Kim TH, Kim YS, et al. Intensity-modulated radiotherapy-based reirradiation for head and neck cancer: a multi-institutional study by Korean Radiation Oncology Group (KROG 1707). Cancer Res Treat 2020;52:1031-40.

11. Choi SH, Chang JS, Choi J, et al. Re-irradiation using intensity-modulated radiotherapy for recurrent and second primary head and neck cancer. Anticancer Res 2018;38:3165-73.

12. Awan MJ, Nedzi L, Wang D, et al. Final results of a multi-institutional phase II trial of reirradiation with concurrent weekly cisplatin and cetuximab for recurrent or second primary squamous cell carcinoma of the head and neck. Ann Oncol 2018;29:9981003.

13. Takiar V, Garden AS, Ma D, et al. Reirradiation of head and neck cancers with intensity modulated radiation therapy: outcomes and analyses. Int J Radiat Oncol Biol Phys 2016;95:1117-31.

14. Bots WT, van den Bosch S, Zwijnenburg EM, et al. Reirradiation of head and neck cancer: long-term disease control and toxicity. Head Neck 2017;39:1122-30.

15. Curtis KK, Ross HJ, Garrett $A L$, et al. Outcomes of patients with loco-regionally recurrent or new primary squamous cell carcinomas of the head and neck treated with curative intent reirradiation at Mayo Clinic. Radiat Oncol 2016;11:1.

16. Lee JY, Suresh $K$, Nguyen $R$, et al. Predictors of severe long-term toxicity after re-irradiation for head and neck cancer. Oral Oncol 2016;60:32-40.

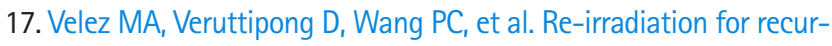
rent and second primary cancers of the head and neck. Oral Oncol 2017;67:46-51.

18. Ohnleiter T, Antoni D, Lefebvre F, et al. Factors improving the outcome of patients re-irradiated with intensity-modulated radiotherapy (IMRT) for relapse or new head and neck cancer developed in irradiated areas. Chin Clin Oncol 2018;7:60.

19. Lee J, Shin IS, Kim WC, Yoon WS, Koom WS, Rim CH. Reirradiation with intensity-modulated radiation therapy for recurrent or secondary head and neck cancer: meta-analysis and systematic review. Head Neck 2020;42:2473-85. 
20. Yard B, Chie EK, Adams DJ, Peacock C, Abazeed ME. Radiotherapy in the era of precision medicine. Semin Radiat Oncol 2015;25: 227-36.

21. Hamoir M, Schmitz S, Suarez $C$, et al. The current role of salvage surgery in recurrent head and neck squamous cell carcinoma. Cancers (Basel) 2018;10:267.

22. Caudell JJ, Ward MC, Riaz N, et al. Volume, dose, and fractionation considerations for IMRT-based reirradiation in head and neck cancer: a multi-institution analysis. Int J Radiat Oncol Biol Phys 2018;100:606-17.

23. Ruhle A, Sprave T, Kalckreuth $T$, et al. The value of moderate dose escalation for re-irradiation of recurrent or second primary headand-neck cancer. Radiat Oncol 2020;15:81.

24. Weichselbaum RR, Beckett MA, Schwartz JL, Dritschilo A. Radioresistant tumor cells are present in head and neck carcinomas that recur after radiotherapy. Int J Radiat Oncol Biol Phys 1988;15:575-9.

25. Nicolay NH, Wiedenmann N, Mix M, et al. Correlative analyses between tissue-based hypoxia biomarkers and hypoxia PET imaging in head and neck cancer patients during radiochemothera- py-results from a prospective trial. Eur J Nucl Med Mol Imaging 2020;47:1046-55.

26. Chopra S, Gupta T, Agarwal JP, Budrukkar A, Ghosh-Laskar S, Dinshaw K. Re-irradiation in the management of isolated neck recurrences: current status and recommendations. Radiother Oncol 2006;81:1-8.

27. Kim J, Kim S, Albergotti WG, et al. Selection of ideal candidates for surgical salvage of head and neck squamous cell carcinoma: effect of the Charlson-age comorbidity index and oncologic characteristics on 1-year survival and hospital course. JAMA Otolaryngol Head Neck Surg 2015;141:1059-65.

28. Richey LM, Shores CG, George J, et al. The effectiveness of salvage surgery after the failure of primary concomitant chemoradiation in head and neck cancer. Otolaryngol Head Neck Surg 2007;136:98-103.

29. Ward MC, Lee NY, Caudell JJ, et al. A competing risk nomogram to predict severe late toxicity after modern re-irradiation for squamous carcinoma of the head and neck. Oral Oncol 2019;90: 80-6. 